\title{
Anti-Tumoral Effect of the Non-Nucleoside DNMT Inhibitor RG108 in Human Pros- tate Cancer Cells
}

\author{
Inês Graça, Elsa Sousa, Tiago Baptista, Mafalda Almeida, João Ramalho-Carvalho, Carlos Palmeira, \\ Rui Henrique and Carmen Jerónimo
}

\begin{abstract}
Background: Current therapeutic strategies for advanced prostate cancer (PCa) are largely ineffective. Because aberrant DNA methylation associated with inappropriate gene-silencing is a common feature of PCa, DNA methylation inhibitors might constitute an alternative therapy. In this study we aimed to evaluate the anti-cancer properties of RG108, a novel non-nucleoside inhibitor of DNA methyltransferases (DNMT), in PCa cell lines.

Methods: The anti-tumoral impact of RG108 in LNCaP, 22Rv1, DU145 and PC-3 cell lines was assessed through standard cell viability, apoptosis and cell cycle assays. Likewise, DNMT activity, DNMTI expression and global levels of DNA methylation were evaluated in the same cell lines. The effectiveness of DNA demethylation was further assessed through the determination of promoter methylation and transcript levels of GSTP1, APC and RAR-/J2, by quantitative methylation-specific PCR and RT-PCR, respectively.

Results: RG108 led to a significant dose and time dependent growth inhibition and apoptosis induction in LNCaP, $22 \mathrm{Rv} 1$ and DU145. LNCaP and 22Rv1 also displayed decreased DNMT activity, DNMT1 expression and global DNA methylation. Interestingly, chronic treatment with RG108 significantly decreased GSTP1, APC and RAR-/J2 promoter hypermethylation levels, although mRNA reexpression was only attained GSTP1 and APC.

Conclusions: RG108 is an effective tumor growth suppressor in most PCa cell lines tested. This effect is likely mediated by reversion of aberrant DNA methylation affecting cancer related-genes epigenetically silenced in PCa. However, additional mechanism might underlie the anti-tumor effects of RG108. In vivo studies are now mandatory to confirm these promising results and evaluate the potential of this compound for PCa therapy.
\end{abstract}

Keywords: Prostate cancer, RG108, DNA methyltransferases, proliferation, apoptosis.

\section{INTRODUCTION}

Prostate cancer $(\mathrm{PCa})$ is the most common diagnosed malignancy in Western countries and one of the leading causes of cancerrelated morbidity and mortality [1]. Although effective therapy is available for early-stage $\mathrm{PCa}$, treatment of advanced disease is mainly ineffective and remains a clinical challenge.

Androgen-deprivation therapy is widely used for locally advanced and systemically spread PCa. However, most patients that initially respond to androgen deprivation therapy have a median time to progression to castration-resistant disease of only 18-30 months [2]. Therefore, investigation of new therapeutic strategies is urgently needed and should be based, ideally, on the acquired knowledge about PCa biology. Indeed, PCa is a complex and heterogeneous disease where multiple genetic and epigenetic alterations interplay and contribute to its development and progression [3]. The most widely known epigenetic change in PCa is aberrant DNA methylation, which may lead to altered expression of tumor suppressor genes and proto-oncogenes. In fact, there are more than 50 genes reported to be silenced by promoter hypermethylation in $\mathrm{PCa}$, including GSTP1, APC, RAR/J2, RASSF1A, and CCND2, among others [4-6]. Remarkably, unlike genetic alterations, epigenetic changes are chemically reversible and numerous compounds have been reported to be effective against cancer cells by inhibiting one or more components of the epigenetic machinery. Among these are the DNA methyltransferases inhibitors (DNMTi), of which 5azacytidine (5-Aza-CR) and 5-aza-2'-deoxycytidine (5-Aza-dCR) are the most widely described [7]. Interestingly, 5-Aza-dCR was shown to have modest clinical activity against castration-resistant $\mathrm{PCa}$ and to inhibit prostate cancer progression in TRAMP mice [8, 9]. However, the effectiveness of these compounds relies on its incorporation into DNA, which may result in substantial, dosedependent cytotoxicity [10]. Owing to the toxicity of nucleoside analogs, emphasis has been placed in the discovery of new compounds that more directly target DNMTs. One of those compounds is RG108, a non-nucleoside analogue of DNMT designed to target human DNMT1 at its active site. RG108 was shown to be effective in human colon cancer cells, in which it was capable of reactivating several tumor suppressors genes (e.g., p16), without affecting the methylation status of centromeric repeats $[11,12]$. Importantly and contrarily to 5-Aza-dCR, the DNA demethylating activity of RG108 was not associated with high levels of citotoxicity [11, 12]. To the best of our knowledge, the anti-tumoral effect of this compound has not been explored in PCa.

Thus, we sought to investigate the potential of RG108 as a growth inhibitor of PCa cells. For that purpose, the impact of RG108 in cell viability, apoptosis and cell cycle of several PCa cell lines was assessed. Furthermore, the inhibitory effect on DNMT and consequent DNA demethylation activity of this drug were evaluated, along with the re-expression of genes known to be epigenetically silenced in PCa. 


\section{MATERIALS AND METHODS}

\section{Prostate Cancer Cell Lines and Drug Preparation}

Prostate cancer cell lines PC-3 and LNCaP were kindly provided by Prof. Ragnhild A. Lothe from the Department of Cancer Prevention at the Institute for Cancer Research, Oslo, Norway, DU145 was obtained from American Type Culture Collection (ATCC, Lockville, MD), and 22Rv1 cells were kindly provided by Dr. David Sidransky at the Johns Hopkins University School of Medicine, Baltimore, MD, USA. LNCaP and 22Rv1 cells were grown in RPMI 1640, DU145 cells were maintained in MEM and PC-3 cells were grown in 50\% RPMI-50\% F-12 medium (GIBCO). All basal culture media were supplemented with $10 \%$ fetal bovine serum and $1 \%$ penicillin/streptomycin (GIBCO). Cells were maintained in an incubator at $37^{\circ} \mathrm{C}$ with $5 \% \mathrm{CO} 2$. All PCa cell lines were karyotyped by G-banding (for validation purposes) and routinely tested for Mycoplasma spp. contamination (PCR Mycoplasma Detection Set, Clontech Laboratories). RG108 was purchased from Tocris Bioscience (Bristol, UK), dissolved in DMSO (Sigma-Aldrich, St. Louis, MO, USA), and stored at $-20^{\circ} \mathrm{C}$ until further use. 5-Aza-dCR was obtained from Sigma (Sigma-Aldrich), dissolved in $50 \%$ acetic acid-50\% PBS and stored at $-20^{\circ} \mathrm{C}$. For control purposes, cell lines were exposed to the vehicles of the drugs only (DMSO or $50 \%$ acetic acid-50\% PBS).

\section{Viability Assay}

PCa cells were seeded at 1000 cells per well onto 96-well flat bottoned culture plates, allowed to adhere overnight and treated with different RG108 concentrations (i.e., 50, 100 and $200 \mu \mathrm{M}$ ) for 3 days (acute exposure) and 14 days (chronic exposure). Cell viability was then evaluated by MTT assay. Briefly, $0.5 \mathrm{mg} / \mathrm{ml}$ of MTT reagent $[3-(4, \quad 5$ dimethylthiazol-2-yl)-2, 5-diphenyl-tetrazolium bromide] was added to each well, and the plates were incubated in the dark for 3 hours at $37^{\circ} \mathrm{C}$. Formazan crystals were then dissolved in DMSO and absorbance was read at $540 \mathrm{~nm}$ in a microplate reader (FLUOstar Omega, BMG Labtech, Offenburg, Germany), subtracting the background, at $630 \mathrm{~nm}$. Three replicates were performed for each condition, and at least two independent experiments were carried out.

\section{Apoptosis Evaluation}

Evaluation of apoptosis was performed using APO Percentage apoptosis assay kit (Biocolor Ltd., Belfast, Northern Ireland) according to the manufacturer's instructions. The assay was carried out using the same cell conditions as those of the MTT assay. Apoptotic cells were assessed at the end of the day 3 and 14 in a FLUOstar Omega microplate reader at $550 \mathrm{~nm}$.

\section{Cell Cycle Analysis}

Cell cycle distribution of PCa cells was determined by flow cytometry. Briefly: $5 \times 10^{5}$ harvested cells were fixed overnight at $4^{\circ} \mathrm{C}$ with $70 \%$ cold ethanol. After washing with cold PBS, cells were ressuspended in staining Propidium Iodide Solution (Cytognos S.L, Salamanca, Spain) and incubated for 30 minutes at room temperature. All cells were then measured on a Cytomics FC500 flow cytometer (Beckman Coulter, Fullerton, CA, USA) and analyzed using Modfit LT (Verity Software House, Inc, Topshan, Maine, USA).

\section{Real Time Quantitative PCR (qRT-PCR)}

RNA was extracted from cell lines using TRIzol® (Invitrogen, Carlsbad, CA) according to manufacturer's instructions. First strand synthesis was performed using the high-capacity CDNA Reverse Transcription Kit from Applied Biosystems (Foster City, CA). Expression of target genes (Ki67, CDKN1A, APC, GSTP1, RAR/J2, DNMT1, DNMT3a, DNMT3b) was quantified using Taqman expression assays, purchased as pre-developed assays from Applied
Biosystems and normalized to the expression of the GUSB housekeeping gene.

\section{Western Blot}

Proteins were extracted from whole-cell lysates using RIPA lysis buffer (Santa Cruz Biotechnology, CA, USA) and subsequently quantified using a Pierce BCA assay (Thermo Scientific Inc., Bremen, Germany), according to the manufacturer's instructions. Briefly, $30 \mu \mathrm{g}$ of protein from each sample were separated using 4-20\% Mini-PROTEAN ${ }^{\circledR}$ TGX $^{\mathrm{TM}}$ Precast Gel at $300 \mathrm{~V}$ and subsequently blotted onto Protran nitrocelulose transfer membranes (Whatman, Dassel, Germany). For immunodetection, membranes were incubated overnight at $4^{\circ} \mathrm{C}$ with antibodies directed against DNMT1 at 1:1,000 and active Caspase-3 at 1:200 (Cell Signaling Technology, Danvers, MA). The Immun-Star WesternC Chemiluminescent kit (Bio-Rad, Hercules, CA, USA) was used to develop the membranes which were then recorded with Amersham Hyperfilm (GE Healthcare Buckinghamshire, UK). To ascertain equal loading of protein, the membranes were incubated with a monoclonal mouse antibody against B-Actin (1:8,000, Sigma-Aldrich). Protein band intensities were determined using Quantity One software (Bio-Rad), by comparing the protein band intensity with the loading control(B-Actin).

\section{DNMT Activity/Inhibition Assay}

Nuclear extracts from vehicle and RG108 treated LNCaP, 22Rv1 and DU145 were obtained using Nuclear Extract Kit (Active Motif, Rixensart, Belgium) as per the manufacture's protocol. After protein quantification, $10 \mu \mathrm{g}$ of nuclear lysate from each sample was used to measure DNMT activity or inhibition with DNMT Activity/Inhibition Assay Kit (Active Motif) according to the manufacturer's instructions. Absorbance was measured using FLUOstar Omega microplate reader, at $450 \mathrm{~nm}$. All samples were analyzed in triplicate.

\section{Quantification of Global DNA Methylation}

Global DNA methylation was analyzed by the Imprint ${ }^{\circledR}$ Methylated DNA Quantification Kit (Sigma-Aldrich) according to the manufacturer's instructions. DNA methylation status was compared with an artificially fully methylated DNA positive control. Absorbance was measured using FLUOstar Omega microplate reader at $450 \mathrm{~nm}$. All samples were analyzed in triplicate.

\section{Quantitative Methylation Specific PCR (qMSP)}

Genomic DNA was extracted from cell lines using a standard technique comprising digestion with proteinase $\mathrm{K}(20 \mathrm{mg} / \mathrm{mL})$ in the presence of $10 \%$ SDS at $55^{\circ} \mathrm{C}$, followed by phenol-chloroform extraction and precipitation with $100 \%$ ethanol [13]. One microgram of DNA was submitted to bisulfite modification using the EZ DNA Methylation-Gold ${ }^{\mathrm{TM}}$ Kit (Zymo Research, Orange, CA) following the manufacturer's instructions. Bisulfite modified DNA was amplified by qMSP using TaqMan technology [14]. Specific GSTP1, $A P C$ and $R A R / J 2$ primers and TaqMan probes were designed using the Methyl Primer Express Software v1.0 (Applied Biosystems). $/ J$ actin $(A C T B)$ was used as an internal reference gene to normalize for DNA input and all qMSP reactions were performed as previously described [4]. Methylation levels for each sample were derived from calibration curves constructed using serial dilutions of bisulfite modified CpGenomeTM Universal Methylated DNA (Millipore, Billerica, MA). GSTP1, APC and $R A R 2 / J$ methylation levels were calculated after normalization for $A C T B$.

\section{Statistical Analysis}

One-way analysis of variance (ANOVA), with post-hoc Dunnet's multiple comparison test when appropriate, was used to compare the results obtained in each parameter for the three different concentrations of RG108 and the controls (vehicle). Analysis was 
performed with SPSS software for Windows version 19.0 (IBMSPSS Inc Chigaco, IL) and statistical significance was set at $P<0.05$.

\section{RESULTS}

\section{RG108 Inhibits Cell Viability and Induces Apoptosis in Human} PCa Cells

To investigate the effects of the demethylating agent RG108 on cell viability, four human PCa cell lines, LNCaP, 22Rv1, DU145 and PC-3 were exposed to three different concentrations of RG108, as wells as to the vehicle of this compound, DMSO. Cell viability was evaluated at days $0,1,2,3,7,10$ and 14. Acute exposure to RG108 resulted in a slight decrease in the number of viable cells (Fig. 1A). Regarding LNCaP, there was a significant decrease in cell viability at the end of the second day when treated with 200 $\mu \mathrm{M}$ of RG108 $(P<0.04)$ compared to the vehicle. $22 \mathrm{Rv} 1$ showed a decrease in cell viability at day 1 when exposed to 50 and $200 \mu \mathrm{M}$ of RG108 $(P<0.04)$ and at the end of the third day when 100 and $200 \mu \mathrm{M}$ concentrations were applied $(P<0.03)$. DU145 treated cells also suffered a slight reduction in viable cells on the first day with 50 and $100 \mu \mathrm{M}$ concentrations $(P<0.02)$ and at day 3 when exposed to $200 \mu \mathrm{M}(P<0.002)$.

As shown in (Fig. 1A), chronic exposure induced an impressive reduction in viable cells especially when the higher concentration of RG108 was used. LNCaP was the most sensitive cell line, since the inhibition of cell growth occurred for all the tested days at all the tested drug concentrations $(P<0.002)$. LNCaP exposure to $200 \mu \mathrm{M}$ RG108 during 14 days resulted in an $80 \%$ reduction in viable cells. $22 \mathrm{Rv} 1$ also displayed a decrease in the number of viable cells in all days $(7,10$, and 14) with $200 \mu \mathrm{M}$ RG108 $(P<0.004)$, being the reduction after 14 days of treatment of $50 \%$, whereas DU145 only showed a decrease of $10 \%$ in cell viability in the last day with 100 and $200 \mu$ M RG108 $(P<0.004)$. RG108 appeared to have no effect on PC-3 cell viability.

To corroborate the previous results, an analysis of molecular changes induced by RG108 was carried out by studying the quantitative expression of two genes involved in cell proliferation pathways. Since the best results were obtained with 14 days of exposure and $200 \mu \mathrm{M}$ of RG108, it was decided to evaluate gene expression under these conditions and at the concentration of $50 \mu \mathrm{M}$. The results showed both induction of $C D K N 1 A(P<0.020)$ and decrease of KI67 $(P<0.045)$ mRNA levels of the RG108 treated cells when compared to the vehicle (Fig. 1B).

To determine if the exposure to RG108 was capable of induce significant cell death, an apoptotic assay that detects the phosphatidylserine transfer to the outside of the membrane was performed. Acute exposure to RG108 resulted in a significant increase in apoptosis (Fig. 1C) for LNCaP with 100 and $200 \mu \mathrm{M}(P<0.037)$ and for $22 \mathrm{Rv} 1$ and DU145 with $100 \mu \mathrm{M}$ (P:S0.002). Chronic treatment induced an augment in apoptosis (Fig. 1C) in LNCaP cells when treated with $100 \mu \mathrm{M}(P<0.038)$ and DU145 when exposed to 50 and $200 \mu \mathrm{M}(P<0.007)$.

To confirm apoptosis at molecular level, the mRNA levels of $C A S P 3$ were evaluated. As the best results achieved with the apoptosis assay were obtained after 14 days, only this time point was evaluated. Chronic treatment of LNCaP $(100 \mu \mathrm{M})$, 22Rv1 $(50 \mu \mathrm{M})$ and DU145 $(200 \mu \mathrm{M})$ resulted in significantly increased levels of CASP3 $(P<0.029)$ (Fig. 1D). An increase of active Caspase 3 protein levels' was observed in all cell lines after drug exposure, namely in LNCaP and DU145 exposed to RG108 at 50 and $200 \mu \mathrm{M}$ and in 22Rv1 when exposed to $100 \mu \mathrm{M}$ (Fig. 1E).

Since RG108 did not affect the cancer phenotype of PC-3 cell line, further studies were only performed in LNCaP, $22 \mathrm{Rv} 1$ and DU145 with 50 and $200 \mu \mathrm{M}$ RG108.
Cell cycle distribution was evaluated after 14 days of exposure to 50 and $200 \mu \mathrm{M}$ RG108. There was no evidence of cell cycle arrest in LNCaP and 22Rv1. However, DU145 presented a significant increase in the percentage of cells in G2/M transition for both concentrations of the compound $(P<0.0001)$ (Fig.1F).

\section{RG108 Inhibits DNMT Activity and Expression in Human PCa Cells}

After exposing cells to 50 and $200 \mu \mathrm{M}$ of RG108 for 14 days, nuclear extracts were obtained and DNMT activity was determined. As observed in (Fig. 2A), there was a significant reduction in DNMT activity (15\%) for 22Rv1 with $50 \mu \mathrm{M}$ RG108 $(P<0.003)$. For the remaining two cell lines a significant decrease in DNMT activity was not apparent, although LNCaP displayed a slight reduction (13\%).

Subsequently, we determined whether decreased DNMT activity correlated with decreased DNMT1, DNMT3a and DNMT3b mRNA levels. As expected, 22Rv1 and LNCaP showed decreased levels of DNMT1 and DNMT3b compared to the vehicle, $22 \mathrm{Rv} 1$ cell line after exposure to $50 \mu \mathrm{M}$ RG108 $(P<0.013)$ and $\mathrm{LNCaP}$ after treatment with both tested concentrations $(P<0.019)$. However, only 22Rv1 demonstrated a significant reduction in DNMT3a mRNA levels $(P<0.001)$ (Fig. 2B). Importantly, a reduction in DNMT1 protein expression was displayed by both cell lines (Fig. 2C).

\section{RG108 Decreases Global DNA Methylation}

Once verified the ability of this compound to decrease DNMT activity and expression, the next step was to investigate the effect in global DNA methylation content. Since DU145 did not present a significant decrease in DNMT activity and expression, it was not further studied. To compare the effect of this demethylating agent with that of 5-Aza-dCR (a widely used demetylating drug), LNCaP and $22 \mathrm{Rv} 1$ cells were exposed to 1 and $5 \mu \mathrm{M}$ of 5 -Aza-dCR during 3 days.

RG108 significantly decreased global DNA methylation in 22Rv1 with $200 \mu \mathrm{M}$ (approximately 5\%, $P<0.028$ ), and in $\mathrm{LNCaP}$ cells (approximately $11 \%$ and $9 \%$ with 50 and $200 \mu \mathrm{M}$ respectively, $P<0.001$ ) (Fig. 3A). In contrast, 5-Aza-dCR was able to reduce global DNA methylation in about $14 \%$ in $\mathrm{LNCaP}$ cells treated with $1 \mu \mathrm{M}(P<0.001)$ and approximately $16 \%$ in $22 \mathrm{Rv} 1$ for both concentrations $(P<0.001)$ (Fig. 3B).

\section{RG108 Causes Demethylation and Reactivation of Silenced Genes in Human PCa Cells}

To investigate the ability to demethylate and consequently induce the expression of specific genes, a panel of three genes $(A P C$, $G S T P 1$ and $R A R / J 2$ ), previously reported to be silenced by promoter hypermethylation in $\mathrm{PCa}$, was evaluated.

Both $\mathrm{LNCaP}$ and $22 \mathrm{Rv} 1$ showed a decrease in methylation levels of GSTP1 with $50 \mu \mathrm{M}(P: S 0.009)$ whereas solely $22 \mathrm{Rv} 1$ exhibited similar results with $200 \mu \mathrm{M}(P<0.005)$ (Fig. 4A). However, only $22 \mathrm{Rv} 1$ demonstrated an increase in GSTP1 mRNA expression with $200 \mu \mathrm{M}(P<0.001)$ (Fig. 4B). APC methylation levels were reduced in $22 \mathrm{Rv} 1$ with $50 \mu \mathrm{M}$ of RG108 $(P<0.005)$ (Fig. 4A), concomitantly with the induction of mRNA expression of this gene $(P<0.003)$ (Fig. 4B). Although a reduction in $R A R / J 2$ methylation was observed in LNCaP with $200 \mu \mathrm{M}(P<0.036)$ and in $22 \mathrm{Rv} 1$ with $50 \mu \mathrm{M}(P<0.003)$ of RG108 (Fig. 4A), the re-expression of this gene was not achieved (Fig. 4B).

The results were also compared with those obtained with 5-aza$\mathrm{dCR}$. This compound was able to demethylate GSTP1 with 1 and 5 $\mu \mathrm{M}$ in $\mathrm{LNCaP}$ and with $1 \mu \mathrm{M}$ in $22 \mathrm{Rv} 1(P<0.020)$. $A P C$ methylation was only reduced in LNCaP cells exposed to $1 \mu \mathrm{M}$ of 5-AzadCR $(P<0.037)$. Concerning $R A R / J 2$, no significant reduction in methylation levels was observed after exposure to 5-Aza-dCR (Fig. 5). 
1A
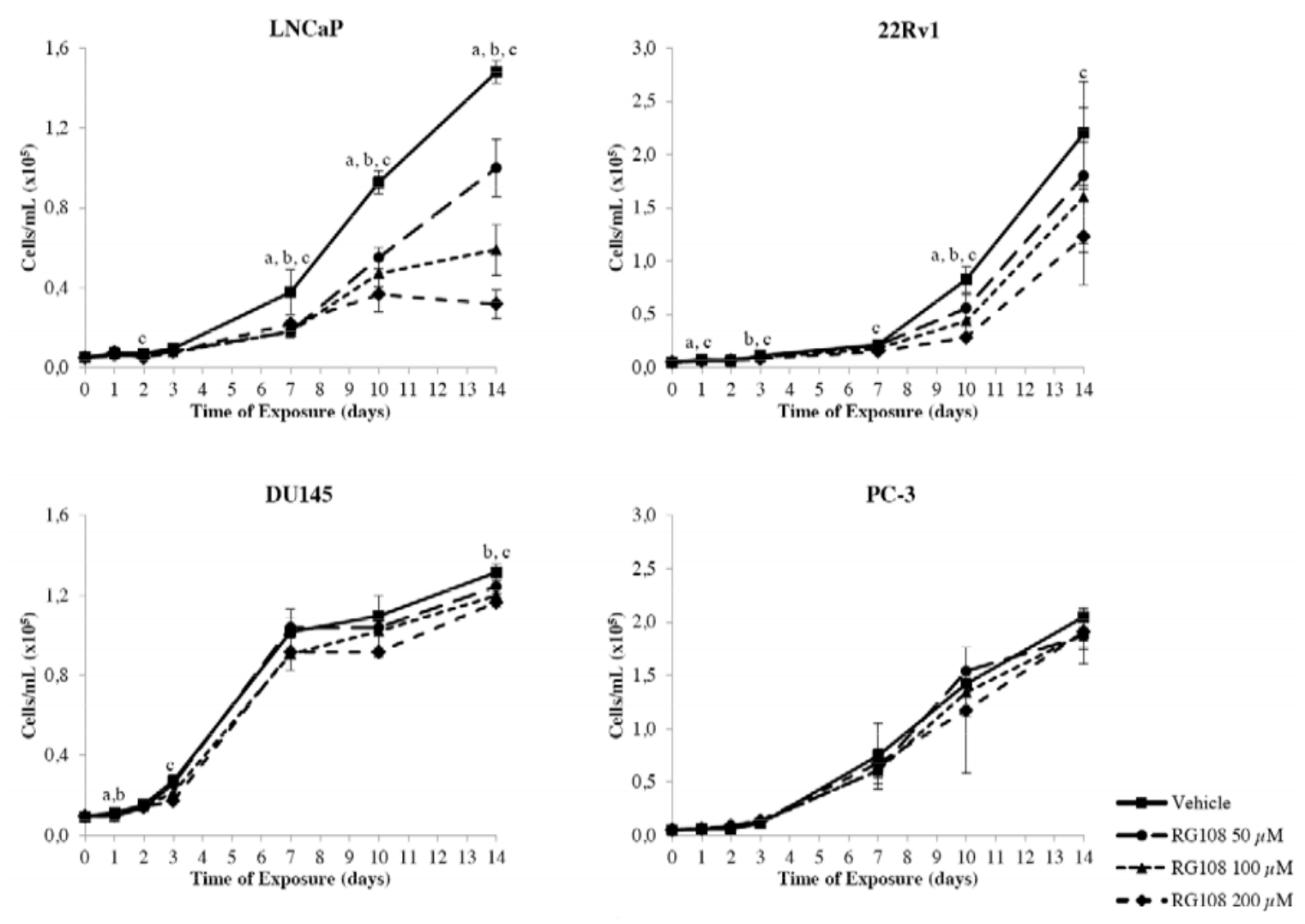

1B
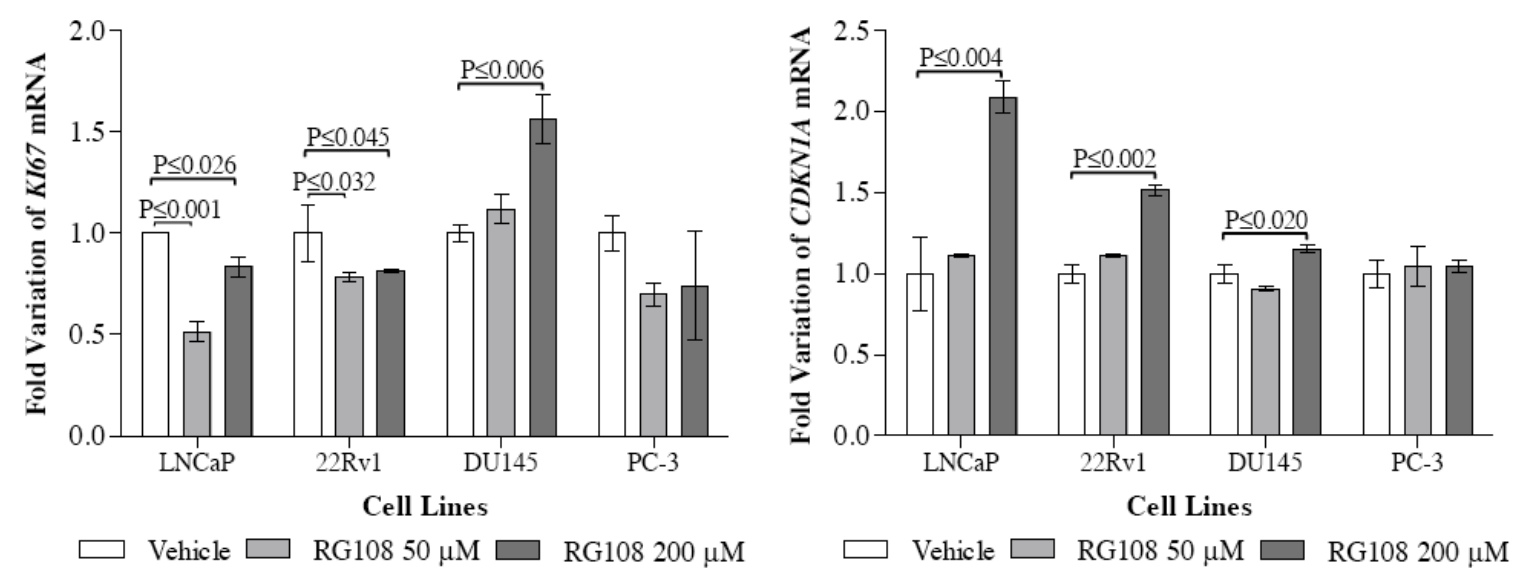

LNCaP

22Rv1

1C
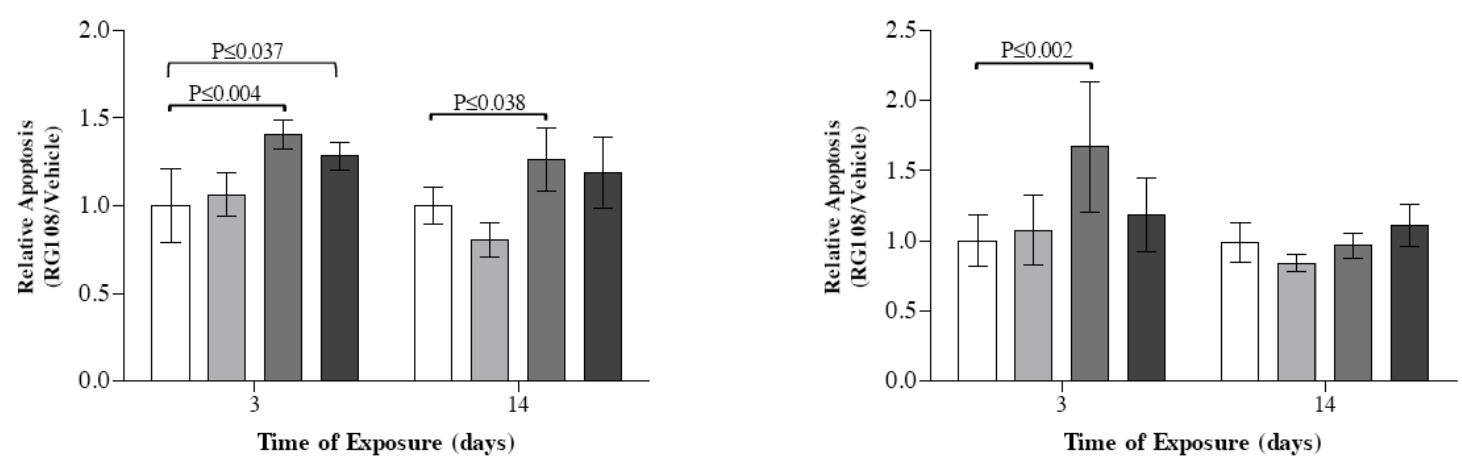

DU145

PC-3 


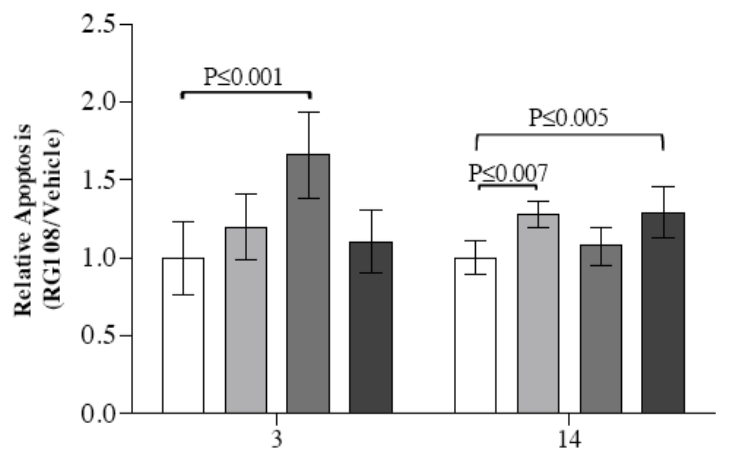

Time of Exposure (days)

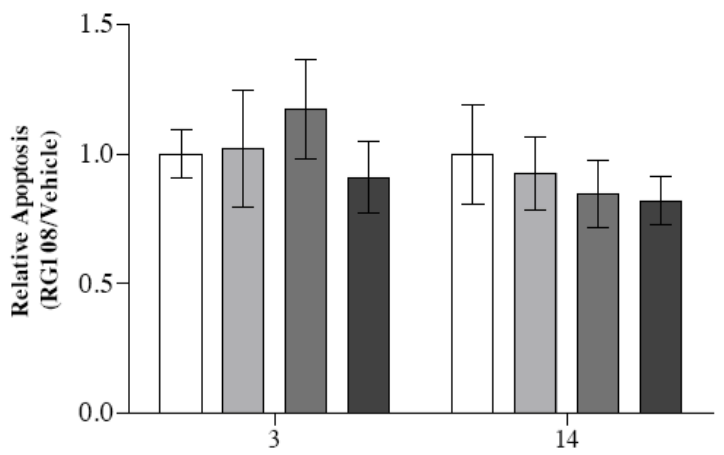

Time of Exposure (days)

$\square$ Vehicle $\square$ RG108 $50 \mu \mathrm{M} \amalg$ RG108 $100 \mu \mathrm{M} \square$ RG108 $200 \mu \mathrm{M}$

1D

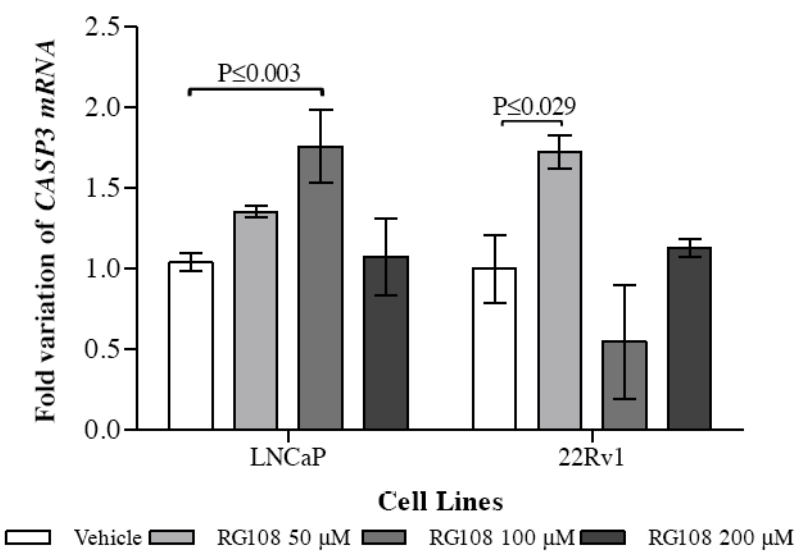



Cell Lines

$\square$ Vehicle $\square$ RG108 $50 \mu \mathrm{M} \square$ RG108 $200 \mu \mathrm{M}$

$1 E$

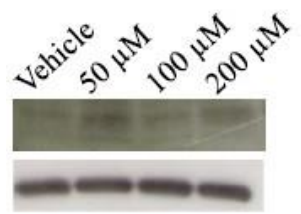

LNCaP

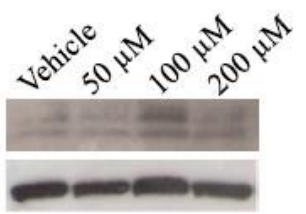

22Rv1

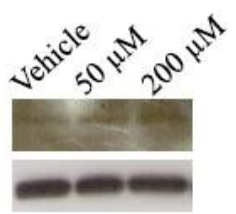

DU145

1F

LNCaP

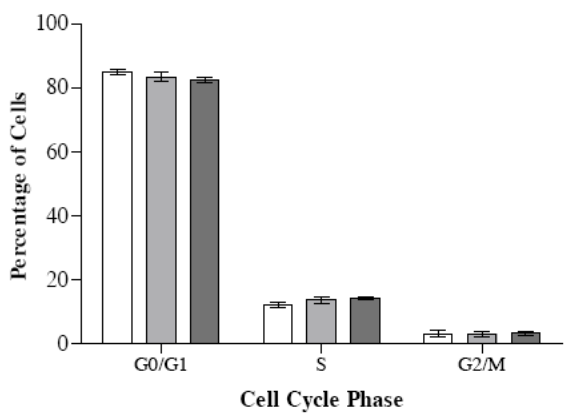

22Rv1

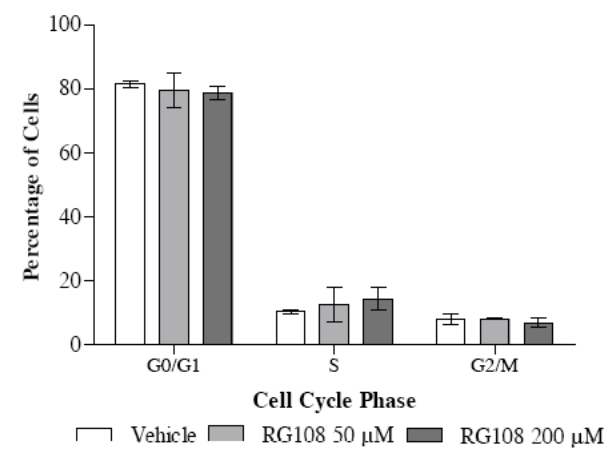

DU145

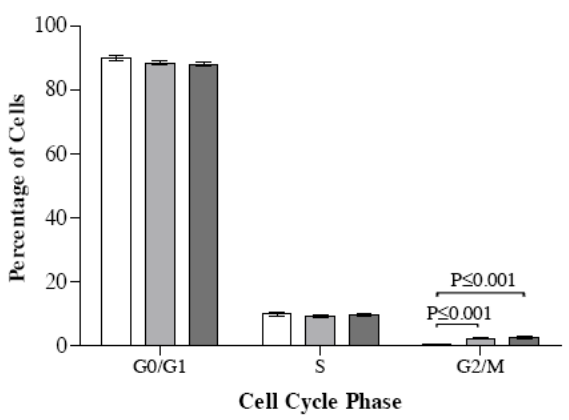

Fig. (1). Effects of RG108 in viability, apoptosis and cell cycle of PCa cell lines. (A) Cell viability in LNCaP, 22Rv1, PC-3 and DU145, in the presence of RG108, at days $0,1,2,3,7,10$, and 14. (a) statistically significant differences were observed between vehicle and $50 \mu \mathrm{M}$ RG108, (b) vehicle and $100 \mu \mathrm{M}$ RG108 and (c) vehicle and $200 \mu \mathrm{M}$ RG108. (B) mRNA expression of Ki67 and CDKN1A after 14 days of exposure to RG108. (C) Effect of acute and chronic RG108 exposure in apoptosis of PCa cell lines. (D) CASP3 mRNA expression and (E) active CASP3 protein expression after 14 days of exposure to RG108. (F) Cell cycle evaluation after 14 days of RG108 exposure. Data are presented as mean of three independent experiments \pm s.d. 


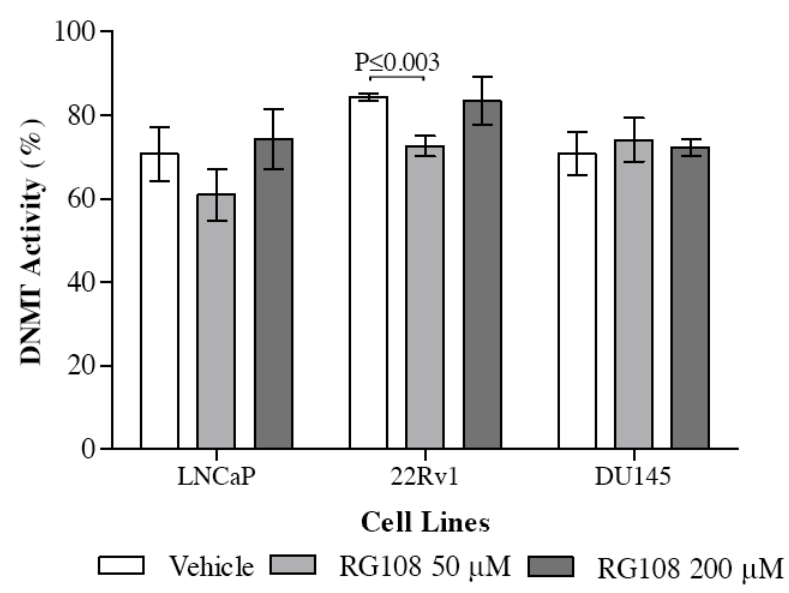

2A
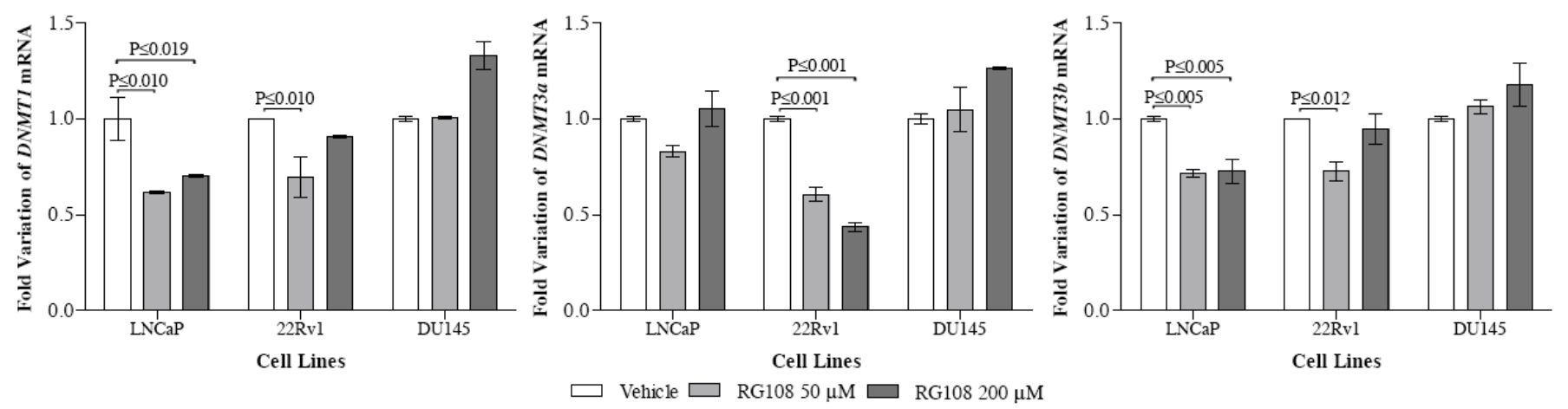

2B

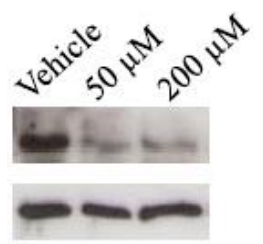

LNCaP

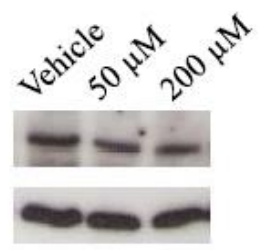

22Rv1

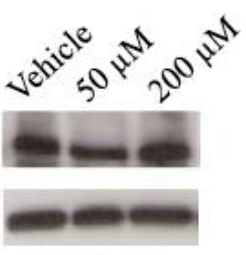

DU145

2C

Fig. (2). Impact of RG108 on DNMT activity (A), in DNMTs mRNA expression (B), and DNMT1 protein expression after 14 days exposure to RG108. Data are shown as mean of three independent experiments \pm s.d.

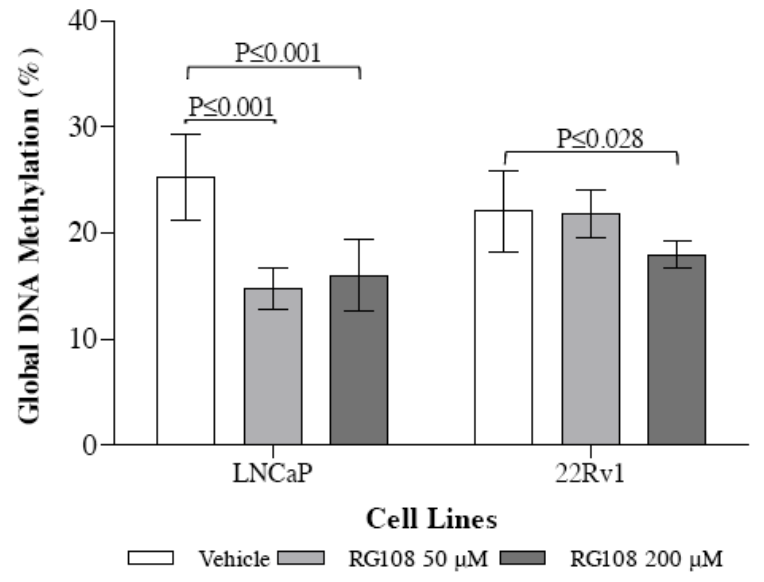

3A

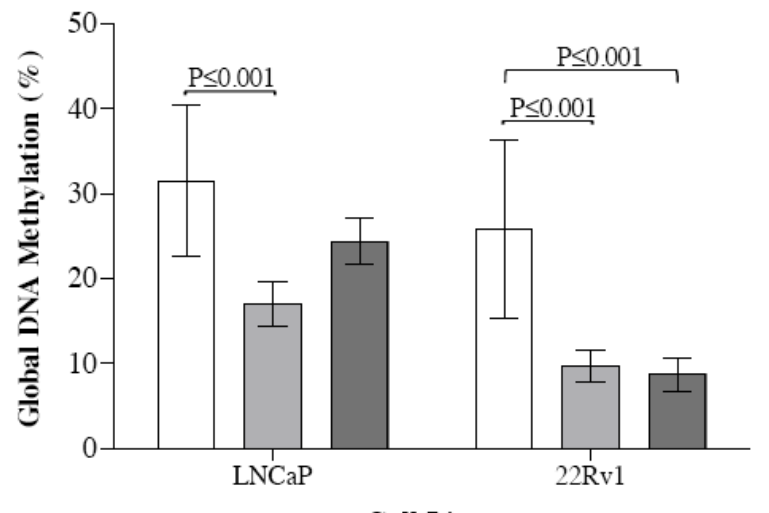

Cell Lines

$\square$ Vehicle $\square$ 5-Aza-2'-deoxycytidine $1 \mu \mathrm{M} \sqcup$ 5-Aza-2'-deoxycytidine $5 \mu \mathrm{M}$ 3B

Fig. (3). Effect of RG108 (A) and 5-aza-CdR (B) on global DNA methylation. Data are presented as mean of three independent experiments \pm s.d. 
Fig. (4A)


Fig. (4B)
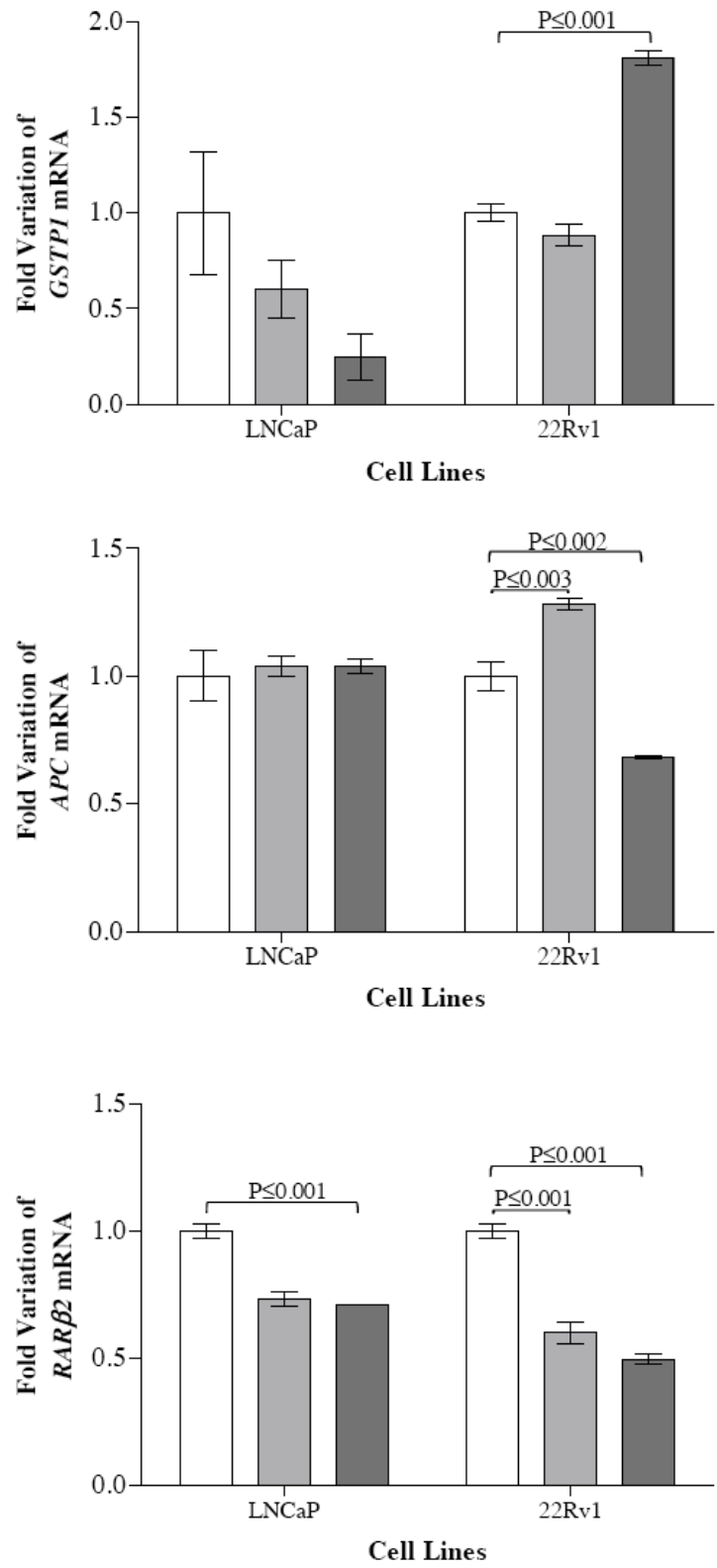

$\square$ Vehicle $\square$ RG108 $50 \mu \mathrm{M} \square$ RG108 $200 \mu \mathrm{M}$

Fig. (4). Impact of RG108 on demethylation (A) and re-expression (B) of GSTP1, APC and RAR/J2. Data are shown as mean of three independent experiments \pm s.d.

\section{DISCUSSION}

The gold standard treatment for hormone dependent PCa involves surgery, radiation or androgen deprivation therapy, depending mainly on disease stage, whereas chemotherapy with docetaxel represents the main therapeutic option for castration-resistant $\mathrm{PCa}$ $[15,16]$. In PCa, as well as in other cancers, the resistance of malignant cells to anticancer agents remains the major cause of treatment failure. Therefore, new therapeutic options are urgently needed. Epigenetic silencing of gene expression, especially that mediated by promoter hypermethylation, plays an important role in the development and progression of $\mathrm{PCa}$, as well as in the emergence of resistance to chemotherapy $[17,18]$. Thus, DNMTs might constitute a valuable therapeutic target for PCa treatment. Reversal of DNA methylation with DNMTi has being widely studied in several cancers. In fact, two nucleoside analogs 5-azacytidine and 5aza-2'-deoxycitidine have been already approved by US Food and Drug Administration (US FDA) for the treatment of myelodysplastic syndrome [19]. However, those two nucleoside analogs have demonstrated a limited efficacy in the treatment of solid malignan- 

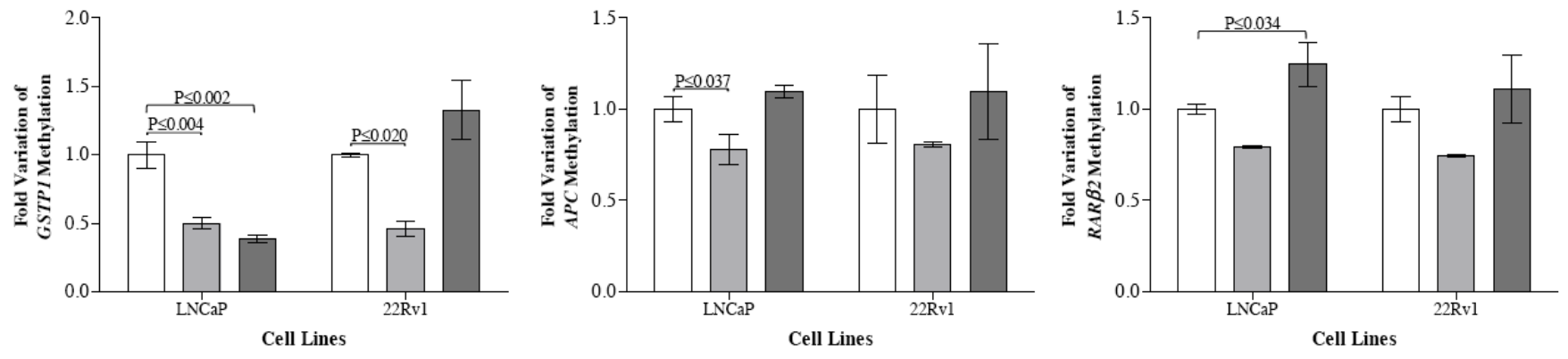

$\square$ Vehicle $\square$ 5-Aza-2'-deoxycytidine $1 \mu \mathrm{M} \square 5$-Aza-2'-deoxycytidine $5 \mu \mathrm{M}$

Fig. (5). Effect of 5-Aza-CdR on GSTP1, APC and RAR/J2 promoter methylation levels. Data are presented as mean of three independent experiments \pm s.d.

cies $[20,21]$. Both for this reason and owing to their significant cytotoxicity, new epigenetic compounds must be explored for cancer treatment.

Herein, we present the first study on the anti-cancer effect of RG108, a non-nucleoside DNMTi, in PCa. The exposure of PCa cell lines (LNCaP, 22Rv1 and DU145) to RG108 resulted in a significant decrease in cell viability, in a dose and time dependent manner, being the $200 \mu \mathrm{M}$ concentration and 14 days (chronic) of exposure the best combination tested. Interestingly, these results emphasize the need to achieve sustained levels of the drug to ensure a prolonged anti-tumor effect, which is in line with current therapeutic guidelines for 5-azacytidine and 5-aza-2'-deoxycitidine in myelodisplastic syndrome [22-24]. Moreover, RG108 induced cell death by apoptosis in LNCaP and DU145, and in the latter, cell cycle arrest in G2/M was also depicted. Importantly, these results were confirmed at the molecular level with a decrease of KI67 transcript levels and induction of $C D K N 1 A$ and $C A S P 3$ mRNA expression. Thus, the ability to disturb multiple regulators of cancer cell survival in these distinct PCa cells lines by RG108 was proven. Because these cell lines are phenotypically different and present diverse patterns of cell response to this drug, it is tempting to suggest that RG108 might be effective against PCa in vivo, which is also characterized by substantial molecular heterogeneity [3].

Some previous studies have reported on the cellular proliferation inhibitory effect of 5-aza-dCR in PCa cell lines [25-27]. In fact, 5 -aza-dCR induces p53 and p21Waf1/Cip1 expression which is associated with inhibition of cell proliferation and induction of apoptosis in LNCaP cells, independently of DNA methylation [28, 29]. In this study we provide evidence that RG108 has similar effects on the inhibition of tumor growth in $\mathrm{PCa}$ cells. Considering that both 5 -aza-dCR and RG108 are effective, the latter might be more appealing from a clinical standpoint, not only because RG108 is a non-nucleoside (and thus may be less cytotoxic), but also because it does not require active cell division to exert its functions [11].

Remarkably, we also demonstrated that RG108 was able not only to inhibit DNMT activity, but also DNMT1, $3 a$ and $3 b$ mRNA expression, as well as DNMT1 protein expression in LNCaP and $22 \mathrm{Rv} 1$ cells. This might justify the observed reversal in GSTP1 and $A P C$ promoter hypermethylation and associated restoration of gene expression. The ability of RG108 to demethylate GSTP1 is a very important finding since this gene, which is methylated in the vast majority of $\mathrm{PCa}$, was recently suggested as a potential useful biomarker for assessing DNMTi efficacy in PCa [30]. These results are also in accordance with the reported ability of RG108 to demethylate tumor suppressor genes (e.g., p16) in HCT116 cell line [11]. It must be recalled, however, that demethylation is not synonymous of re-expression, as clearly demonstrated in our study for $R A R / J 2$. Because epigenetic silencing of cancer-related genes is a more complex phenomenon, in which other mechanisms, such as histone post-translational modifications, are also involved [17], combined therapies with histone deacetylase inhibitors might prove to be more effective.

This study also showed that RG108 has a weaker demethylating effect compared to 5-aza-dCR, as three days exposure to the latter was able to more effectively reduce the levels of global DNA methylation than RG108 at 14 days. Similar results were reported for HCT116 cells, which showed a reduction of $50 \%$ of cytosine methylation levels when exposed to 5-Aza-dCR (after five days of incubation) and of only 30\% when exposed to RG108 (after 15 days) [11]. The superior efficacy of 5aza-dCR might be due to its properties as a cytidine analogue. Once incorporated into the DNA, 5azadCR covalently traps the DNMTs on the DNA by forming a suicide complex which can lead to a massive loss of DNA methylation, resulting in genome hypomethylation, which may predispose to genomic instability and foster neoplastic transformation of normal cells [23, 31]. RG108, on the other hand, directly blocks DNMT1 activity [11], and may, thus, explain the less potent global DNA demethylation effect. This therapeutical disadvantage of RG108 may, however, be compensated by a lesser degree of global hypomethylation, minimizing genomic instability and improving its toxicity and safety profile.

Interestingly, several studies have proposed 5-Aza-dCR as an adjuvant to conventional therapy of $\mathrm{PCa}[25,26,32,33]$. Those studies demonstrated that 5 -Aza-dCR could sensitize PCa cell lines to docetaxel, which is the first line therapy for castration-resistant PCa [25]. Likewise, 5-Aza-dCR might restore the expression of androgen receptor in PC-3 cell line, and the combined treatment with bicalutamide demonstrated a synergistic effect in repressing tumor growth in xenograft mice [32]. Remarkably, bicalutamide was recently associated with higher DNMT3a and DNMT3b expression, potentiating the up-regulation of truncated androgen receptor isoforms and therefore the castration resistant phenotype [33]. However, when cells were exposed to 5-Aza-dCR, there was a decrease in DNMT activity and a consequent decline in the development of the castration-resistant phenotype [33]. Because RG108 is able to modify the phenotype of PCa cells and the catalytic domain of the four DNMTs is highly conserved, predicting similar interactions of those enzymes with RG108, it would be interesting to evaluate the possible synergistic action between RG108 and conventional PCa therapy. Moreover, based on the promising results from this study, further experiments should be conducted to investigate the cellular pathways involved in RG108 response. Comparing these cellular pathways with those involved in 5-Aza-dCR response might provide valuable information concerning $\mathrm{PCa}$ cells biology and illuminate the different mechanisms of action of these compounds.

\section{CONCLUSIONS}

The demethylating agent RG108 demonstrated the ability to reduce cell viability, induce cellular death by apoptosis, and decrease DNMT activity and DNMT1 expression in PCa cells. Fur- 
thermore, it was able to decrease promoter hypermethylation levels and induce re-expression of genes known to be epigenetically silenced in PCa. Because RG108 performed better in chronic exposure (14 days) at $200 \mu \mathrm{M}$ concentration, further studies should be conducted with these conditions to further assess the promising role of RG108 as a valuable tool for PCa therapy.

\section{CONFLICT OF INTEREST}

The authors confirm that this article content has no conflicts of interest.

\section{ACKNOWLEDGEMENTS}

IG and ES performed and interpreted the phenotypic and molecular assays. IG, RH and CJ contributed to experimental design and wrote the manuscript with input from co-authors. IG and CP performed cell cycle analysis. TB, MA and JCR assisted on cell culture and drug exposure assays. The authors would like to express gratitude to the Laboratory of Flow Cytometry at the Department of Hematology of Portuguese Oncology Institute-Porto.

This study was funded by research grants from Liga Portuguesa Contra o Cancro - Núcleo Regional do Norte, Research Center of Portuguese Oncology Institute - Porto (CI-IPOP 4-2008) and European Community's Seventh Framework Programme - Grant number FP7-HEALTH-F5-2009-241783. IG was supported by a FCTFundação para a Ciência e a Tecnologia grant (SFRH/BD/64082/ 2009), ES was supported by a grant from Liga Portuguesa Contra o Cancro - Núcleo Regional do Norte and MA and JRC were supported by the European Community's Seventh Framework Programme Grant.

\section{REFERENCES}

[1] Siegel R, Naishadham D, Jemal A. Cancer statistics, 2012. CA: a cancer J Clinicians 2012; 62(1): 10-29.

[2] Kohli M, Tindall DJ. New developments in the medical management of prostate cancer. Mayo Clinic proceedings Mayo Clinic 2010; 85(1): 77-86.

[3] Shen MM, Abate-Shen C. Molecular genetics of prostate cancer: new prospects for old challenges. Genes Development 2010; 24(18): 1967-2000.

[4] Jeronimo C, Henrique R, Hoque MO, et al. A quantitative promoter methylation profile of prostate cancer. Clinical cancer research : an official J Am Assoc Cancer Res 2004; 10(24): 8472-8.

[5] Henrique R, Costa VL, Cerveira N, et al. Hypermethylation of Cyclin D2 is associated with loss of mRNA expression and tumor development in prostate cancer. J Mol Med 2006; 84(11): 911-8.

[6] Jeronimo C, Henrique R, Hoque MO, et al. Quantitative RARbeta2 hypermethylation: a promising prostate cancer marker. Clinical cancer research : an official J Am Assoc Cancer Res 2004; 10(12 Pt 1): 4010-4.

[7] Lyko F, Brown R. DNA methyltransferase inhibitors and the development of epigenetic cancer therapies. J Natl Cancer Inst 2005; 97(20): 1498-506.

[8] Thibault A, Figg WD, Bergan RC, et al. A phase II study of 5-aza2 'deoxycytidine (decitabine) in hormone independent metastatic (D2) prostate cancer. Tumori 1998; 84(1): 87-9.

[9] McCabe MT, Low JA, Daignault S, et al. Inhibition of DNA methyltransferase activity prevents tumorigenesis in a mouse model of prostate cancer. Cancer Res 2006; 66(1): 385-92.

[10] Juttermann R, Li E, Jaenisch R. Toxicity of 5-aza-2'-deoxycytidine to mammalian cells is mediated primarily by covalent trapping of DNA methyltransferase rather than DNA demethylation. Proc Natl Acad Sci USA 1994; 91(25): 11797-801.

[11] Brueckner B, Garcia Boy R, Siedlecki P, et al. Epigenetic reactivation of tumor suppressor genes by a novel small-molecule inhibitor of human DNA methyltransferases. Cancer Res 2005; 65(14): 6305-11.

[12] Bardenheuer W, Lehmberg K, Rattmann I, et al. Resistance to cytarabine and gemcitabine and in vitro selection of transduced cells after retroviral expression of cytidine deaminase in human hematopoietic progenitor cells. Leukemia : official J Leukemia Soc Am, Leukemia Res Fund, UK 2005; 19(12): 2281-8.

[13] Pearson H, Stirling D. DNA extraction from tissue. Methods Mol Biol 2003; 226: 33-4.

[14] Eads CA, Danenberg KD, Kawakami K, et al. MethyLight: a highthroughput assay to measure DNA methylation. Nucleic acids Res 2000; 28(8): E32.

[15] Nakabayashi M, Sartor O, Jacobus $S$, et al. Response to docetaxel/carboplatin-based chemotherapy as first- and second-line therapy in patients with metastatic hormone-refractory prostate cancer. BJU international 2008; 101(3): 308-12.

[16] Galsky MD, Small AC, Tsao CK, Oh WK. Clinical development of novel therapeutics for castration-resistant prostate cancer: Historic Challenges and Recent Successes. CA: a Cancer J Clinicians 2012.

[17] Jeronimo C, Bastian PJ, Bjartell A, et al. Epigenetics in prostate cancer: biologic and clinical relevance. Eur Urol 2011; 60(4): 75366.

[18] Mishra MV, Bisht KS, Sun L, et al. DNMT1 as a molecular target in a multimodality-resistant phenotype in tumor cells. Molecular cancer research : MCR 2008; 6(2): 243-9.

[19] Abdulhaq H, Rossetti JM. The role of azacitidine in the treatment of myelodysplastic syndromes. Expert Opinion Investigational Drugs 2007; 16(12): 1967-75.

[20] Appleton K, Mackay HJ, Judson I, et al. Phase I and pharmacodynamic trial of the DNA methyltransferase inhibitor decitabine and carboplatin in solid tumors. J clinical oncology : official J Am Soc Clin Oncol 2007; 25(29): 4603-9.

[21] Bauman J, Verschraegen C, Belinsky S, et al. A phase I study of 5azacytidine and erlotinib in advanced solid tumor malignancies. Cancer Chemotherapy Pharmacol 2012; 69(2): 547-54.

[22] Kaminskas E, Farrell AT, Wang YC, Sridhara R, Pazdur R. FDA drug approval summary: azacitidine (5-azacytidine, Vidaza) for injectable suspension. The oncologist 2005; 10(3): 176-82.

[23] Jones PA, Taylor SM. Cellular differentiation, cytidine analogs and DNA methylation. Cell 1980; 20(1): 85-93.

[24] Kantarjian H, Issa JP, Rosenfeld CS, et al. Decitabine improves patient outcomes in myelodysplastic syndromes: results of a phase III randomized study. Cancer 2006; 106(8): 1794-803.

[25] Festuccia C, Gravina GL, D'Alessandro AM, et al. Azacitidine improves antitumor effects of docetaxel and cisplatin in aggressive prostate cancer models. Endocrine-Related Cancer 2009; 16(2): 401-13.

[26] Gravina GL, Festuccia C, Millimaggi D, et al. Chronic azacitidine treatment results in differentiating effects, sensitizes against bicalutamide in androgen-independent prostate cancer cells. The Prostate 2008; 68(7): 793-801.

[27] Festuccia C, Gravina GL, Biordi L, et al. Effects of EGFR tyrosine kinase inhibitor erlotinib in prostate cancer cells in vitro. The Prostate 2009; 69(14): 1529-37.

[28] Pulukuri SM, Rao JS. Activation of p53/p21Waf1/Cip1 pathway by 5aza-2'-deoxycytidine inhibits cell proliferation, induces proapoptotic genes and mitogen-activated protein kinases in human prostate cancer cells. Int J Oncol 2005; 26(4): 863-71.

[29] Bott SR, Arya M, Kirby RS, Williamson M. p21WAF1/CIP1 gene is inactivated in metastatic prostatic cancer cell lines by promoter methylation. Prostate Cancer Prostatic Diseases 2005; 8(4):321-6.

[30] Chiam K, Centenera MM, Butler LM, Tilley WD, Bianco-Miotto T. GSTP1 DNA methylation and expression status is indicative of 5 -aza-2'-deoxycytidine efficacy in human prostate cancer cells. PloS one 2011; 6(9): e25634.

[31] Gabbara S, Bhagwat AS. The mechanism of inhibition of DNA (cytosine-5-)-methyltransferases by 5-azacytosine is likely to involve methyl transfer to the inhibitor. Biochemical J 1995; 307 ( Pt 1): 87-92.

[32] Gravina GL, Marampon F, Di Staso M, et al. 5-Azacitidine restores and amplifies the bicalutamide response on preclinical models of androgen receptor expressing or deficient prostate tumors. The Prostate 2010; 70(11): 1166-78.

[33] Gravina GL, Marampon F, Piccolella M, et al. Hormonal therapy promotes hormone-resistant phenotype by increasing DNMT activity and expression in prostate cancer models. Endocrinology 2011; 152(12): 4550-61. 